Pacific Journal of Mathematics

MODULARITY OF THE CONGRUENCE LATTICE OF A
COMMUTATIVE CANCELLATIVE SEMIGROUP 


\title{
MODULARITY OF THE CONGRUENCE LATTICE OF A COMMUTATIVE CANCELLATIVE SEMIGROUP
}

\section{HowaRD HamiLtoN}

\begin{abstract}
Modularity of the lattice of normal subgroups of a group is well-known. Equivalently, the lattice of congruence relations on a group is a modular lattice. A natural question to consider is how far can we push the last statement when dealing with the larger class semigroups. It is easily shown that the class of congruence lattices of semigroups satisfies no nontrivial lattice identity. Thus we might try to find those semigroups whose congruence lattice is a modular lattice. This problem is of all the more interest due to the fact that congruences on algebras whose congruence lattice is a modular lattice satisfy variants of the Jordan-Holder-Schreier theorem. In this paper we show that the commutative cancellative semigroups whose congruence lattice is a modular lattice are the abelian groups, the positive cones of rational groups, and the nonnegative cones of rational groups. We also show that the commutative cancellative semigroups with a distributive lattice of congruences are locally cyclic or locally cyclic with an identity adjoined. This last result generalizes Ore's theorem that a group has a distributive lattice of congruences if and only if it is locally cyclic.
\end{abstract}

1. Introduction. By an $N$-semigroup we mean a commutative cancellative archimedean semigroup without idempotent. An $\bar{N}$ semigroup will denote a commutative cancellative idempotent free semigroup (CCIF-semigroup) which contains an ideal which is an $\mathrm{N}$ semigroup.

It was shown in 1957 by Tamura [14] (see also [1] and [13]) that every $N$-semigroup $S$ can be represented by an abelian group $G$ and a function $I$ from $G \times G$ into the nonnegative integers $N^{0}$ with the properties

$$
\begin{aligned}
& \quad I(g, h)=I(h, g) \text { for all } g, h \in G, \\
& \text { (1.2) } I(g, h)+I(g h, k)=I(g, h k)+I(h, k) \text { for all } g, h, k \in G, \\
& \text { (1.3) } I(g, e)=1 \text { for all } g \in G \text {, where } e \text { is the identity of } G, \\
& \text { (1.4) For each } g \in G \text { there exists } m>0 \text { such that } I\left(g, g^{m}\right)>0 \text {. }
\end{aligned}
$$

Where $S=N^{0} \times G$ with the product

$$
(m, g)(n, h)=(m+n+I(g, h), g h) \text { for }(m, g),(n, h) \in N^{0} \times G .
$$


We write $S=(G, I)$ if $S$ is determined in this manner by $G$ and $I$. Condition (1.1) is the requirement on $I$ that makes the product (1.5) commutative, while (1.2) gives associativity of (1.5). Property (1.3) is just a normalizing condition. Condition (1.4) is the requirement that makes $(G, I)$ an archimedean semigroup. Cancellation in $\left(N^{\circ},+\right)$ and $G$ makes $(G, I)$ cancellative, also. Thus we see that if we were to drop condition (1.4) all we would lose, possibly, is the archimedeanness of $S$. In [9] it is shown that dropping (1.4) yields an $\bar{N}$-semigroup and that every $\bar{N}$-semigroup can be obtained in this way. [9] also contains further information on the structure of $\bar{N}$ semigroups and CCIF-semigroups. We will need the following information about $\bar{N}$-semigroups from [9].

Lemma 1.1. [9] Let $S$ be an $\bar{N}$-semigroup. Let $T$ be the $N$ semigroup ideal of $S$. Then

(i) $T=\{a \in S \mid$ for all $b \in S$ there exist $m>0$ and $c \in S$ such that $\left.a^{m}=b c\right\}$.

(ii) For each $a \in T \rho_{a}$ is a group congruence on $S$ where for $x, y \in S$ we have $(x, y) \in \rho_{a}$ if there exists $m, n>0$ such that $a^{m} x=a^{n} y$.

(iii) If $S=(G, I)$ then for all $g \in G$ we have $(1, g) \in T$ and $(0, e \in T$ if $e$ is the identity of $G$.

Let $S=\mathrm{U}_{\alpha \in \Gamma} S_{\alpha}$, where $\Gamma$ is a lower semilattice, be the greatest semilattice decomposition of a semigroup $S$. For each $\alpha \in \Gamma$ the $\alpha$ filter of $S$ is $S^{\alpha}=\bigcup_{\beta \geqq \alpha} S_{\beta}$. Then $S$ is the $\Gamma$ direct limit of $S^{\alpha}$.

Fact 1.2. If $S$ is a CCIF-semigroup then the $\alpha$-filters of $S$ are all $\bar{N}$-semigroups.

Next we will discuss a relationship between congruences on a semigroup and congruences on the $\alpha$-filters of the semigroup. Later we will apply this to CCIF-semigroups.

For a semigroup $X$ we will let $L(X)$ denote the lattice of all congruence relations on $X$. If $\sigma_{\alpha} \in L\left(S^{\alpha}\right)$ for some $\alpha \in \Gamma$ then $\sigma^{\alpha}=$ $\sigma_{\alpha} \cup\left(S \backslash S^{\alpha}\right) \times\left(S \backslash S^{\alpha}\right)$ is a congruence on $S$, and the map $\sigma_{\alpha} \mapsto \sigma^{\alpha}$ is an isomorphism of $L\left(S^{\alpha}\right)$ onto a sublattice of $L(S)$. Thus we have

Lemma 1.3. For each $\alpha \in \Gamma L\left(S^{\alpha}\right)$ is isomorphic to a sublattice of $L(S)$.

Next, let $\mathscr{L}=\left\{\prod_{\alpha} \sigma_{\alpha} \in \prod_{\alpha} L\left(S^{\alpha}\right)\right.$ : for $\beta, \gamma \in \Gamma$ with $\left.\beta \geqq \gamma \sigma_{\beta} \subseteq \sigma_{\gamma}\right\}$.

Lemma 1.4. $\mathscr{L}$ is a complete sublattice of $\Pi_{\alpha} L\left(S^{\alpha}\right)$.

Proof. Let $\left\{\rho_{\delta}=\prod_{\alpha} \sigma_{\alpha, \delta}: \delta \in \Lambda\right\}$ be a collection of members of $\mathscr{L}$. 
Then by the definition of join in the direct product $\Pi_{\alpha} L\left(S^{\alpha}\right)$ we have $\mathrm{V}_{\delta \in \Lambda} \rho_{\delta}=\prod_{\alpha}\left(\mathrm{V}_{\delta \in \Lambda} \sigma_{\alpha, \delta}\right)$, and $\mathrm{V}_{\delta \in \Lambda} \sigma_{\beta, \delta} \subseteq \mathrm{V}_{\delta \in \Lambda} \sigma_{\gamma, \delta}$ if $\beta \geqq \gamma$ because $\sigma_{\beta, \delta} \subseteq \sigma_{\gamma, \delta}$ for all $\delta$ if $\beta \geqq \gamma$. Hence $\mathrm{V}_{\delta} \rho_{\delta} \in \mathscr{L}$. Similarly, one sees that $\bigcap_{\delta} \rho_{\delta}=\Pi_{\alpha}\left(\bigcap_{\delta \in \Lambda} \sigma_{\alpha, \delta}\right)$ is in $\mathscr{L}$.

Define a function $f: \mathscr{L} \rightarrow L(S)$ by $f\left(\Pi_{\alpha} \sigma_{\alpha}\right)=\bigcup_{\delta} \sigma_{\alpha}$. Note that $\bigcup_{\alpha} \sigma_{\alpha} \in L(S)$ because of the definition of $\mathscr{L}$.

THEOREM 1.5. The map $f$ is a closed lattice homomorphism of $\mathscr{L}$ onto $L(S)$.

Proof. Let $\Pi_{\alpha} \sigma_{\alpha}$ and $\Pi_{\alpha} \tau_{\alpha}$ be two members of $\mathscr{L}$.

$$
f\left(\prod_{\alpha} \sigma_{\alpha} \vee \prod_{\alpha} \tau_{\alpha}\right)=f\left(\prod_{\alpha}\left(\sigma_{\alpha} \vee \tau_{\alpha}\right)\right)=\bigcup_{\alpha}\left(\sigma_{\alpha} \vee \tau_{\alpha}\right),
$$

and $f\left(\Pi_{\alpha} \sigma_{\alpha}\right) \vee f\left(\Pi_{\alpha} \tau_{\alpha}\right)=\left(\mathbf{U}_{\alpha} \sigma_{\alpha}\right) \vee\left(\mathbf{U}_{\alpha} \tau_{\alpha}\right)$. Since $\mathbf{U}_{\alpha} \sigma_{\alpha}$ and $\mathbf{U}_{\delta} \tau_{\alpha}$ are both contained in $\bigcup_{\alpha}\left(\sigma_{\alpha} \vee \tau_{\alpha}\right)$, we have immediately that $\mathbf{U}_{\alpha}\left(\sigma_{\alpha} \vee \tau_{\alpha}\right) \supseteqq\left(\mathbf{U}_{\alpha} \sigma_{\alpha}\right) \vee\left(\mathbf{U}_{\alpha} \tau_{\alpha}\right)$. To see containment the other direction, let $(a, b) \in \bigcup_{\alpha}\left(\sigma_{\alpha} \vee \tau_{\alpha}\right)$ then there exists $\beta \in \Gamma$ such that $(a, b) \in \sigma_{\beta} \vee \tau_{\beta}$. That is, there exists $\beta \in \Gamma$ and $a=a_{0}, a_{1}, a_{2}, \cdots$, $a_{n}=b \in S^{\beta}$ such that $\left(a_{i}, a_{i+1}\right) \in \sigma_{\beta}$ or $\left(a_{i}, a_{i+1}\right) \in \tau_{\beta}$ for $i=0,1, \cdots$, $n-1$. Thus $(a, b) \in\left(\bigcup_{\alpha} \sigma_{\alpha}\right) \vee\left(\mathbf{U}_{\alpha} \tau_{\alpha}\right)$, and we have shown that $\mathbf{U}_{\alpha}\left(\sigma_{\alpha} \vee \tau_{\alpha}\right) \leqq\left(\mathbf{U}_{\alpha} \sigma_{\alpha}\right) \vee\left(\mathbf{U}_{\alpha} \tau_{\alpha}\right)$. Therefore, $f$ preserves joins.

Now we show that $f$ preserves intersections. We have

$$
f\left(\prod_{\alpha} \sigma_{\alpha} \cap \prod_{\alpha} \tau_{\alpha}\right)=f\left(\prod_{\alpha}\left(\sigma_{\alpha} \cap \tau_{\alpha}\right)\right)=\bigcup_{\alpha}\left(\sigma_{\alpha} \cap \tau_{\alpha}\right),
$$

and $f\left(\Pi_{\alpha} \sigma_{\alpha}\right) \cap f\left(\Pi_{\alpha} \tau_{\alpha}\right)=\left(\mathbf{U}_{\alpha} \sigma_{\alpha}\right)$. Since $\mathbf{U}_{\alpha}\left(\sigma_{\alpha} \cap \tau_{\alpha}\right)$ is contained in each of $\mathbf{U}_{\alpha} \sigma_{\alpha}$ and $\mathbf{U}_{\alpha} \tau_{\alpha}$ we have $\mathbf{U}_{\alpha}\left(\sigma_{\alpha} \cap \tau_{\alpha}\right) \cong\left(\mathbf{U}_{\alpha} \sigma_{\alpha}\right) \cap\left(\mathbf{U}_{\alpha} \tau_{\alpha}\right)$. Now let $(a, b) \in\left(\mathbf{U}_{\alpha} \sigma_{\alpha}\right) \cap\left(\mathbf{U}_{\alpha} \tau_{\alpha}\right)$. That is, $(a, b) \in \mathbf{U}_{\alpha} \sigma_{\alpha}$ and $(a, b) \in$ $\bigcup_{\alpha} \tau_{\alpha}$. Hence there exist $\beta, \gamma \in \Gamma$ such that $(a, b) \in \sigma_{\beta}$ and $(a, b) \in \tau_{\gamma}$, and since $\Pi_{\alpha} \sigma_{\alpha}$ and $\Pi_{\alpha} \tau_{\alpha}$ are in $\mathscr{L}$ we have $(a, b) \in \sigma_{\beta r}$ and $(a, b) \in \tau_{\beta \gamma}$. Thus $(a, b) \in\left(\sigma_{\beta \gamma} \cap \tau_{\beta \gamma}\right)$ and so $(a, b) \in \bigcup_{\alpha}\left(\sigma_{\alpha} \cap \tau_{\alpha}\right)$. Therefore $f$ preserves intersections.

From Lemmas 1.2 and 1.4 and Theorem 1.5 we immediately have

THEOREM 1.6. Let $S$ be a semigroup. $L(S)$ satisfies an identity if aud only if $L\left(S^{\alpha}\right)$ satisfies the same identity for each $\alpha \in \Gamma$.

If $\rho$ is a binary relation on a set $X$ and $Y \subseteq X$ then $\rho \mid Y=$ $\rho \cap(Y \times Y)$. Also, $\iota_{X}$ will denote the identity relation on $X$, and $\omega_{X}$ will denote the universal relation on $X$.

Theorem 1.7. ([19] Proposition 3.1) If $\rho$ is a group congruence 
on $S$ and $J$ is an ideal of $S$ then $\sigma=\rho \mid J$ is a group congruence on $J$ and $S / \rho \cong J / \sigma$. Furthermore, $\rho$ is the unique extension of $\sigma$ to a group congruence on $S$.

COROLLARY 1.8. The join semilattice of group congruences on a semigroup $S$ is isomorphic onto the join semilattice of group congruences on any ideal $J$ of $S$.

Lemma 1.9. Let $S$ be a semigroup and let $J$ be an ideal of $S$. Let $\sigma$ be a group congruences on $J$ and $\mu$ the unique extension of $\sigma$ to a group congruence on $S$. Let $\eta=\sigma \cup \iota_{S}$ and let $\tau$ be any group congruence on $S$. Then $\eta \in L(S)$ and $\eta \vee \tau=\mu \vee \tau$.

Proof. $\sigma=\mu \mid J$ implies that $\eta$ is a congruence relation on $S$. We have $\eta \subseteq \mu$ therefore $\eta \vee \tau \subseteq \mu \vee \tau$. We also have $(\mu \vee \tau) \mid J=$ $(\mu \mid J) \vee(\tau \mid J)=\sigma \vee(\tau \mid J) \leqq(\eta \vee \tau) \mid J$ where the first "=" follows from Corollary 1.8. Now $\eta \vee \tau$ and $\mu \vee \tau$ are group congruences on $S$ with $(\mu \vee \tau)|J \subseteq(\eta \vee \tau)| J$. Hence again by Corollary $1.8 \eta \vee \tau \supseteqq$ $\mu \vee \tau$. Thus $\eta \vee \tau=\mu \vee \tau$, and we are done.

DEFINITION 1.1. A semigroup $S$ is a proper subdirect product of two semigroups $T$ and $U$ if $S$ is a subdirect product of $T$ and $U$ and $S \not T$ and $S \not U$. In which case it follows that there exist nontrivial congruences $\mu$ and $\nu$ on $S$ such that $\mu \cap \nu=\iota_{S}$.

CoROllary 1.10. If a semigroup $S$ is a proper subdirect product of two groups and if $S$ has a proper ideal $J$ then $L(S)$ is not modular.

Proof. Let $S$ be a proper subdirect product of two groups then there exist nontrivial congruences $\mu$ and $\tau$ on $S$ such that $S / \mu$ and $S / \tau$ are groups and $\mu \cap \tau=\iota_{S}$. Let $\eta=(\mu \mid J) \cup \iota_{S}$. By Lemma 1.9 $\eta \in L(S)$ and $\eta \vee \tau=\mu \vee \tau$. And since $\eta \cong \mu$ we have $\eta \cap \tau=\iota_{S}$. Hence $L(S)$ contains

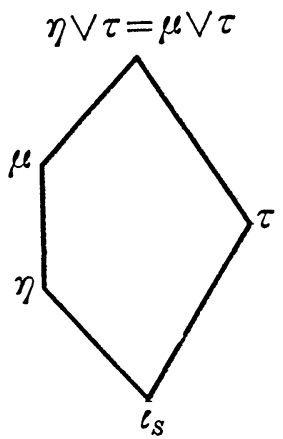


as a sublattice; so $L(S)$ is not a modular lattice.

\section{CCIF-semigroups with $L(S)$ modular.}

Definition 2.1. An $N$-semigroup $S$ is power-joined if for all $a, b \in S$ there exist $m$ and $n$ greater that zero such that $a^{m}=b^{n}$.

From [8] we have the following theorem about power-joined $N$-semigroups.

THEOREM 2.2. Let $S$ be an $N$-semigroup. Then the following are equivalent:

(a) $S$ is power-joined.

(b) $S$ is not a subdirect product of two groups.

(c) The group congruences on $S$ form a sublattice of $L(S)$.

Corollary 2.3. Let $S$ be an $N$-semigroup. If $L(S)$ is modular then $S$ is power-joined.

This is immediate from Theorem 2.2 and Corollary 1.10 because being a subdirect product of two groups is equivalent to being a proper subdirect product of two groups for semigroups which are not groups.

Let $S=(G, I)$ be an $N$-semigroup. If $|G|=1$ then $S \cong N$, the positive integers with addition. In [2], Exercise 6, p. 137, it is shown that $L\left(N^{0}\right)$ is a distributive lattice. Let $\rho \in L(N)$ then $\rho^{*}=$ $\rho \cup\{(0,0)\} \in L\left(N^{0}\right)$ and $\rho \mapsto \rho^{*}$ is an embedding of $L(N)$ into $L\left(N^{0}\right)$. Thus $L(N)$ is distributive and hence modular.

For an $N$-semigroup $S$ we will introduce the following notation: $L_{n}(S)$ is the collection of nil congruences on $S, L_{r}(S)$ is the collection of Rees congruences on $S$, and $L_{g}(S)$ is the collection of group congruences on $S$. In general $L_{n}(S)$ and $L_{r}(S)$ are both sublattices of $L(S)$, and by Theorem $2.2 L_{g}(S)$ is a sublattice of $L(S)$ if and only if $S$ is power-joined.

Lemma 2.4. Let $S=(G, I)$ be an $N$-semigroup. If $L(S)$ is modular then $L_{n}(S)=L_{r}(S)$.

Proof. We can assume that $|G|>1$ because otherwise $S \cong N$ from the above remarks and it is clear for $N$ that $L_{n}(N)=L_{r}(N)$. If $|G|>1, L(S)$ is modular and $\eta \in L_{n}(S) \backslash L_{r}(S)$ then let $I_{\eta}$ be the ideal of $S$ which is the zero of $S / \eta$. Let $\rho_{I_{\eta}}$ be the Rees congruence associated with $I_{\eta}$, then $\rho_{I_{\eta}} \varsubsetneqq \eta$. Let $\tau$ be the group congruence on $S$ corresponding to the homomorphism $\pi$ of $S$ onto $G$ given by $\pi(m, g)=g$ 
for all $(m, g) \in S$. Since the join of any group congruence with any nil congruence is $\omega_{S}$, we have $\eta \vee \tau=\rho_{I_{\eta}} \vee \tau=\omega_{S}$. Let $\sigma=\tau \mid I$. If $(m, g)$ and $(n, g)$ are in $S \backslash I_{\eta}$ and $((m, g),(n, g)) \in \eta$ with $m>n$ then since $(m, g)=(n, g)(0, e)^{m-n}$ so $[(n, g)]_{\eta}$ divides $[(n, g)]_{\eta}$ in $S / \eta$, but in a nil semigroup $x y=x$ implies $x=0$. Thus we have a contradiction, and so $((m, g),(n, h)) \in \eta$ for $(m, g)$ and $(n, h) \in S \backslash I_{\eta}$ implies $g \neq h$. Hence we have $\eta \cap \tau=\rho_{I_{\eta}} \cap \tau=\sigma \cup \iota_{S}$. Thus we have

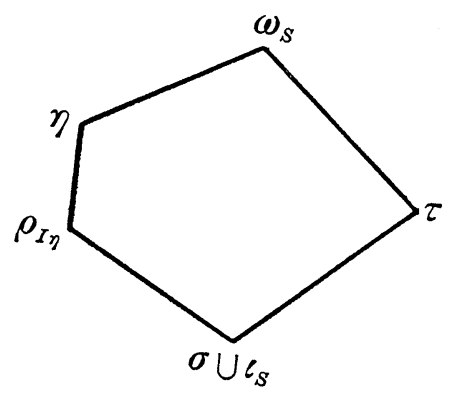

contained in $L(S)$, and so $L(S)$ is not a modular lattice. Thus $L_{n}(S)=$ $L_{r}(S)$.

Tamura has classified the commutative archimedean semigroups in the following four classes:

Type (i ) commutative nil semigroups.

Type (ii) commutative nil extensions of abelian groups.

Type (iii) $N$-semigroups.

Type (iv) commutative archimedean nonpotent noncancellative semigroups.

Since a homomorphic image of an archimedean semigroup is also archimedean, a homomorphic image of an $N$-semigroup $S$ has one of the four types above. Let $\sigma \in L(S)$ then we call $\sigma$ a nil- $(n g-, N-, T 4-)$ congruence on $S$ if $S / \sigma$ is of Type i (ii, iii, iv). Dickinson [5] has noted that a T4-congruence is a refinement of an $\mathrm{N}$-congruence.

Definition 2.5. An $N$-semigroup $S$ is said to be irreducible if $\iota_{S}$ is the only $N$-congruence on $S$.

Tamura [15] has shown that an irreducible $N$-semigroup is isomorphic onto a positive real additive semigroup. It is also shown 
in [15] that every $N$-semigroup $S=(G, I)$ is a subdirect product of an irreducible $N$-semigroup and the structure group $G$ of $S$.

Lemma 2.6. Let $S$ be an $N$-semigroup. If $L_{n}(S)=L_{r}(S)$ then $S$ is irreducible.

Proof. Suppose $S$ is not irreducible then $S$ is a proper subdirect product of an irreducible (hence positive real) $N$-semigroup $T$ and an abelian group $G$. Let $\pi_{T}: S \rightarrow T$ be the projection of $S$ onto $T$ (we identify $S$ with its isomorphic image in $T \times G)$. We have $\pi_{r}$ is not one-to-one because the subdirect decomposition is proper. Thus $(m, g)$ and $(m, h)$ are in $S$ for some $m \in T$ and $g, h \in G$ with $g \neq h$. Let $J=$ $\{(n, k) \in S: n>m$ and $k \in G\}$ then $J$ is an ideal of $S$. Define a relation $\eta$ on $S$ by $\eta=\omega_{J} \cup\{((m, g),(m, h)),((m, h),(m, g))\}$. Then $\eta$ is a nilcongruence on $S$ which is not a Rees congruence. Thus $L_{n}(S) \neq L_{r}(S)$.

Definition 2.7. Let $S$ be a semigroup with zero. Then an element $x \in S$ such that $x y=y x=0$ for all $y \in S$ is called an annihilator of $S$. The collection of annihilators of $S$ is denoted by $A(S)$.

DeFinition 2.8. Let $S$ be a commutative semigroup. If $x$ and $y$ are in $S$ then we say $x$ divides $y$ in $S$ (denoted $x \mid y$ or $\left.x\right|_{s} y$ ) if there exists $z \in S$ such that $x z=y$.

THeOREM 2.9. (Tamura [17]) Let $S$ be an $N$-semigroup. Then the cancellative congruences on $S$ form a sublattice of $L(S)$ isomorphic onto the lattice of subgroups of the group of quotients of $S$. Hence the cancellative congruences on an $N$-semigroup form a modular lattice.

LEMmA 2.10. Let $S$ be a power-joined $N$-semigroup such that $L_{n}(S)=L_{r}(S)$. Let $\sigma \in L(S)$. If $\sigma \neq i$ then there exists $\tau \in L_{g}(S)$ and $\eta \in L_{r}(S)$ such that $\sigma=\tau \cap \eta$ and $\tau$ and $\eta$ are uniquely determined by $\sigma$.

Proof. By Lemma 2.6 $S$ is an irreducible $N$-semigroup. Therefore, from Dickinson's result we know that $S$ has no T4-congruences. Hence if $\sigma \neq i$ then $\sigma$ is either a Rees congruence of an $n g$-congruence. In either case $S / \sigma$ contains a group ideal (in the case $\sigma$ is a nil-congruence the group is the trivial group). Let $J_{\sigma}$ denote the ideal of $S$ which maps onto this group ideal under the natural homomorphism of $S$ onto $S / \sigma$. Then $\sigma \mid J_{\sigma}$ is a group congruence on $J_{\sigma}$. By Theorem 1.7 let $\tau$ be the group congruence on $S$ which extends $\sigma \mid J_{\sigma}$. Let $\eta$ be 
the Rees congruences $\bmod J_{\sigma}$. Clearly $\tau \cap \eta=\sigma \mid J_{\sigma} \cup \iota_{S}$. Since $J_{\sigma} / \sigma$ is an ideal of $S / \sigma,(S / \sigma) /\left(J_{\sigma} / \sigma\right)$ is a nil semigroup. Let $\nu$ be the congruence on $S$ such that $S / \nu \cong(S / \sigma) /\left(J_{o} / \sigma\right)$. Then $\nu \in L_{n}(S)=L_{r}(S)$. But $\nu=\omega_{J_{\sigma}} \cup \sigma \mid\left(S \backslash J_{\sigma}\right)$ so $\sigma \mid\left(S \backslash J_{\sigma}\right)$ must be $\iota_{\left(S \backslash J_{\sigma}\right)}$. Hence $\sigma=$ $\sigma \mid J_{\sigma} \cup \iota_{S}=\tau \cap \eta$.

Uniqueness of $\tau$ and $\eta$ : Suppose $\tau_{1}, \tau_{2} \in L_{g}(S)$ and $\eta_{1}, \eta_{2} \in L_{r}(S)$ and that $\tau_{1} \cap \eta_{1}=\tau_{2} \cap \eta_{2}$. Let $I_{\eta_{i}}$ be the ideal of $S$ associated with $\eta_{i}(i=1,2)$. Suppose $I_{\eta_{1}} \neq I_{\eta_{2}}$. Assume $x \in I_{\eta_{1}} \backslash I_{\eta_{2}}$ then by the proof of Theorem 1.7 there is a $y \in I_{\eta_{1}} \cap I_{\eta_{1}}$ such that $(x, y) \in \tau_{1}$. Therefore $(x, y) \in \tau_{1} \cap \eta_{1}$, but $(x, y) \notin \eta_{2}$ so $(x, y) \notin \tau_{2} \cap \eta_{2}$ This contradicts the assumption that $\tau_{1} \cap \eta_{1}=\tau_{2} \cap \eta_{2}$. Therefore $I_{\eta_{1}} \subseteq I_{\eta_{2}}$. Similarly we get $I_{\eta_{2}} \subseteq I_{\eta_{1}}$. Hence $\eta_{1}=\eta_{2}$. And so $\tau_{1}\left|I_{\eta_{1}}=\tau_{2}\right| I_{n_{1}}$ and so again using Theorem 1.7 we have $\tau_{1}=\tau_{2}$. This proves the uniqueness of $\tau$ and $\eta$.

We are now ready to prove the theorem determining the $\mathrm{N}$ semigroups whose congruence lattice is modular.

THEOREM 2.11. Let $S$ be a power-joined $N$-semigroup. Then the following are equivalent:

(1) $L(S)$ is a modular lattice.

(2) $L_{n}(S)=L_{r}(S)$.

(3) For each ideal $J$ of $S$ we have $|A(S / J)| \leqq 2$.

(4) Divisibility is a total order on $S$.

(5) $S$ is isomorphic to a positive cone of an additive subgroup of the rationals.

In which case $L(S) \cong\left(L_{g}(S) \times L_{r}(S)\right)^{1}$ (where $L^{1}$ means a smallest element 1 is adjoined to the lattice $L)$.

Proof. We show $(1) \Leftrightarrow(2),(2) \Rightarrow(3) \Rightarrow(4) \Rightarrow(2)$, and $(4) \Leftrightarrow(5)$. $(1) \Rightarrow(2)$ is shown in Lemma 2.4 .

Proof of $(2) \Rightarrow(1)$. Assume that $S$ is a power-joined $N$-semigroup for which $L_{n}(S)=L_{r}(S)$. Define a map $\lambda$ from $L(S)$ into $\left(L_{g}(S) \times L_{r}(S)\right)^{1}$ by

$$
\lambda(\sigma)=\left\{\begin{array}{lll}
1 & \text { if } & \sigma=i \\
(\tau, \eta) & \text { if } & \sigma \neq i \text { and } \sigma=\tau \cap \eta \text { for } \tau \in L_{g}(S) \text { and } \eta \in L_{r}(S) .
\end{array}\right.
$$

From Lemma 2.10 it follows that $\lambda$ is well-defined and $\lambda^{-1}(\tau, \eta)=$ $\tau \cap \eta, \lambda^{-1}(1)=i$ is the inverse of $\lambda$.

$\lambda$ is order preserving. Let $\sigma_{1} \subseteq \sigma_{2}$ for $\sigma_{1}, \sigma_{2} \in L(S)$. If $\lambda\left(\sigma_{1}\right)=1$ 
then $\lambda\left(\sigma_{1}\right) \leqq \lambda\left(\sigma_{2}\right)$ since 1 is the smallest element of $\left(L_{g}(S) \times L_{r}(S)\right)^{1}$. Thus assume that $\lambda\left(\sigma_{1}\right) \neq 1$. Then $\lambda\left(\sigma_{i}\right)=\left(\tau_{i}, \eta_{i}\right)$ for $i=1,2$ with $\sigma_{i}=\tau_{i} \cap \eta_{i}(i=1,2)$. Let $I_{\eta_{i}}$ denote the ideal of $S$ associated with $\eta_{i}(i=1,2)$. If $I_{\eta_{1}} \nsubseteq I_{\eta_{2}}$ choose $x \in I_{\eta_{1}} \backslash I_{\eta_{2}}$ and $y \in I_{\eta_{1}} \cap I_{\eta_{2}}$ such that $(x, y) \in \tau_{1}$. Then $(x, y) \in \tau_{1} \cap \eta_{1}=\sigma_{1} \subseteq \sigma_{2}=\tau_{2} \cap \eta_{2}$ which implies that $(x, y) \in \eta_{2}$, but this is a contradiction, since $x \notin I_{\eta_{2}}$ and $y \in I_{\eta_{2}}$. Thus $I_{\eta_{1}} \subseteq I_{\eta_{2}}$ and so $\eta_{1} \subseteq \eta_{2}$. Now $\sigma_{1} \subseteq \sigma_{2}$ implies $\sigma_{1}\left|I_{\eta_{1}} \subseteq \sigma_{2}\right| I_{\eta_{1}}$, than is $\tau_{1} \mid I_{\eta_{1}} \subseteq I_{\eta_{1}}$ (see the proof of Lemma 2.10). Hence by Theorem 1.7 and Corollary $1.8 \tau_{1} \leqq \tau_{2}$. Thus $\left(\tau_{1}, \eta_{1}\right) \leqq\left(\tau_{2}, \eta_{2}\right)$ and $\lambda$ is order preserving.

It is obvious that $\lambda^{-1}$ is also order preserving. Hence $\lambda$ is a closed lattice isomorphism of $L(S)$ onto $\left(L_{g}(S) \times L_{r}(S)\right)^{1}$. In our present case (i.e., $S$ is power-joined) we have $L_{g}(S)$ is a sublattice of $L(S)$ by Theorem 2.2 and so by Theorem $2.9 L_{g}(S)$ is a modular lattice. $L_{r}(S)$ is always a modular lattice, since it is isomorphic onto a sublattice of the Boolean lattice of all subsets of $S$ with inclusion as the partial order. The isomorphism here is just the one taking $\eta \in L_{r}(S)$ to its associated ideal of $S$. Thus $L_{g}(S) \times L_{r}(S)$ being the direct product of modular lattices is a modular lattice. But adjoining a smallest element to a modular lattice also gives a modular lattice. Hence $L(S)$ is a modular lattice. This completes the proof that $(2) \Rightarrow(1)$.

Proof of $(2) \Longrightarrow(3)$. Let $L_{n}(S)=L_{r}(S)$ and choose $J$ an ideal of $S$. If $u, v \in S$ are two distinct nonzero annihilators of $S / J$, then $\rho=\omega_{J} \cup$ $\{(u, v),(v, u)\}$ is in $L_{n}(S)$ but not in $L_{r}(S)$. A contradiction. Hence $|A(S / J)| \leqq 2$.

Proof of $(3) \Rightarrow(4)$. Note that for an $N$-semigroup divisibility is always a partial order. Assume $|A(S / J)| \leqq 2$ for every ideal $J$ of $S$. If there exists $u, v \in S$ such that $u \nmid v$ and $v \nmid u$, then let $J=$ $(u S) \cup(v S)$. Then $A(S / J) \supseteqq\{u, v, 0\}$. This contradicts our assumption, thus either $u \mid v$ or $v \mid u$ and $S$ is totally ordered by divisibility.

Proof of $(4) \Longrightarrow(2)$. Let $S$ be an $N$-semigroup in which divisibility is a total order. Tamura [15] has shown that such semigroups are irreducible and are therefore isomorphic into the positive reals. The assumption that $S$ be power-joined puts us into the positive rationals (see Tamura and Sasaki [20]) with addition understood to be the operation in all of our real semigroups. Thus we will assume that $S$ is contained in the positive rationals. Let $J$ be an ideal of $S$. If $x \in J$ and $y \in S$ with $y>x$ then $x \mid y$ hence $y \in J$. Thus $J$ is a segment of $S$ (i.e., $J=(c, \infty)_{S}=\{x \in S: x>c\}$ or $J=[c, \infty)_{S}=\{x \in S: x \geqq c\}$ 
for some rational $c \geqq 0)$. Let $\eta \in L_{n}(S)$ and let $J_{\eta}$ denote the associated ideal of $S$. Let $c=\inf \left(J_{\eta}\right)$ and suppose $a, b \in S$ with $a<b$ and $(a, b) \in \eta$ then $(a, b),(b, b+(b-a)), \cdots,(b+(m-1)(b-a), b+m(b-a))$ are all in $\eta$. And for $m$ large enough $b+m(b-a)>c$ hence $b+m(b-a) \in J_{\eta}$ and so $a, b \in J_{\eta}$. Thus $\eta \in L_{r}(S)$. This completes the proof of $(4) \Rightarrow(2)$.

The equivalence of (4) and (5) is easily seen when we realize that (4) puts us into the positive rationals as mentioned in the proof of (4) implies (2).

We are now ready to answer the question of modularity of the congruence lattice of a CCIF-semigroup.

THeOREM 2.12. Let $S$ be a CCIF-semigroup. Then $L(S)$ is a modular lattice if and only if $S$ is a positive cone of a subgroup of the additive rationals.

Proof. Let $S=\bigcup_{\alpha \in \Gamma} S_{\alpha}$ be the greatest semilattice decomposition of $S$. All we need to do to is show that each $\alpha$-filter $S^{\alpha}$ is an $N$-semigroup if $L(S)$ is a modular lattice. Assume that $L(S)$ is a modular lattice. Then by Theorem $1.6 L\left(S^{\alpha}\right)$ is modular for each $\alpha \in \Gamma$. Choose $\alpha \in \Gamma$. By Fact $1.2 S^{\alpha}$ is an $\bar{N}$-semigroup. Let $S^{\alpha}=(G, I)$ for some abelian group $G$. We will be done when we show that $G$ must be a torsion abelian group because then $I$ must satisfy condition (1.4) and so $S^{\alpha}$ is an $N$-semigroup. Suppose $G$ contains an element $g_{0}$ of infinite order. From Lemma 1.1 we see that $\left(1, g_{0}\right)$ and $(0, e)$, where $e$ is the identity of $G$, are in the $N$ semigroup ideal of $S$ and so by part (ii) of Lemma 1.1 the relations $\rho\left(1, g_{0}\right)$ and $\rho(0, e)$ are group congruences on $S$. Suppose $(m, g)$ and $(n, h)$ are in $S$ then $((m, g),(n, h)) \in \rho_{(0, e)}$ if and only if $g=h$, and $((m, g),(n, g)) \in \rho_{\left(1, g_{0}\right)}$ implies that $m=n$ since $g_{0}$ has infinite order. Thus $\rho_{\left(1, g_{0}\right)} \cap \rho_{(0, e)}=i$ and so $S^{\alpha}$ is a subdirect product of the two nontrivial groups $\left.S^{\alpha} / \rho_{\left(1, g_{0}\right.}\right)$ and $S^{\alpha} / \rho(0, e)$. Therefore by Corollary 1.10 we have a contradiction to the assumption that $L\left(S^{\alpha}\right)$ is a modular lattice. Hence $G$ is a torsion abelian group and we are done.

3. Commutative cancellative semigroups with idempotents with $L(S)$ modular. In this section we will determine those commutative cancellative semigroups with an idempotent (which is necessarily an identity element of the semigroup), which are not groups, whose congruence lattice is a modular lattice.

Let $S=\mathbf{U}_{\alpha \in \Gamma} S_{\alpha}$ be the greatest semilattice decomposition of a commutative cancellative semigroup with an identity element. Then $\Gamma$ has a maximal element $\alpha_{0}$ and $S_{\alpha_{0}}$ is an abelian group. Assume 
that $L(S)$ is a modular lattice and assume $S \neq S_{\alpha_{0}}$ (i.e., $S$ is not an abelian group). By Theorem $1.6 L\left(S^{\alpha}\right)$ is modular for all $\alpha \in \Gamma$. Also, if $\alpha \neq \alpha_{0}$ then $S^{\alpha}$ contains $S_{\alpha}$, which is an $N$-semigroup, as an ideal; hence, we have

Lemma 3.1. For each $\alpha \neq \alpha_{0}, S_{\alpha}$ is a power-joined $N$-semigroup.

Proof. Assume that $\alpha \neq \alpha_{0}$ and $S_{\alpha}$ is not power-joined then by Theorem 2.2 $S_{\alpha}$ is a subdirect product of two groups. Equivalently, there are two group congruences $\sigma$ and $\tau$ on $S_{\alpha}$ such that $\sigma \cap \tau=\iota_{S_{\alpha}}$. Let $\bar{\sigma}$ and $\bar{\tau}$ be the unique extensions of $\sigma$ and $\tau$, respectively, to group congruences on $S^{\alpha}$. Suppose $(x, y) \in \bar{\sigma} \cap \bar{\tau}$, and choose $z \in S_{\alpha}$. Then $(x z, y z) \in \bar{\sigma} \cap \bar{\tau}$ with $x z, y z \in S_{\alpha}$. Therefore $(x z, y z) \in \sigma \cap \tau=\iota$, and so $x z=y z$. Thus $x=y$, since $S$ is cancellative, and we have $\bar{\sigma} \cap \bar{\tau}=\iota_{S^{\alpha}}$. Hence by Corollary 1.10 we have $L\left(S^{\alpha}\right)$ is not modular. This is a contradiction and therefore $S_{\alpha}$ must be power-joined.

In [7] Theorem 2.5 Hall showed that for a power-joined $N$ semigroup $A$ the translation semigroup $T(A)$ of $A$ is a commutative cancellative semigroup having two archimedean components. One component is an ideal containing the inner translations of $A$ (and hence a copy of $A$ ) and the other component is the group of permutation translations of $A$ and is isomorphic to a subgroup of any structure group of $A$. By [20] every structure group of a powerjoined $N$-semigroup is a torsion abelian group. Hall also showed that a commutative cancellative ideal extension of $A$ is isomorphic to a subsemigroup of $T(A)$.

LeMma 3.2. $|\Gamma|=2$.

Proof. Suppose $|\Gamma|>2$. Then there exist $\alpha, \beta \in \Gamma$ such that $\beta<\alpha<\alpha_{0}$. By Lemma $3.1 S_{\beta}$ is a power-joined $N$-semigroup. Thus from Hall's work we have $S^{\beta}$ is isomorphic to a subsemigroup of $T\left(S_{\beta}\right)$ containing the copy of $S_{\beta}$. We will identify $S_{\beta}$ and $S^{\beta}$ with their images in $T\left(S_{\beta}\right)$. Let $T\left(S_{\beta}\right)=A \cup B$ be the greatest semilattice decomposition of $T\left(S_{\beta}\right)$. Let $A$ denote the archimedean component containing $S_{\beta}$ and let $B$ denote the archimedean component consisting of the group of permutation translations of $S_{\beta}$. Let $x \in S_{\alpha}$. If $x \in B$ then, since $B$ is torsion group, there exists $m>0$ such that $x^{m}=1$ so $1 \in S_{\alpha}$. But $1 \in S_{\alpha_{0}}$, a contradiction. If $x \in A$ then $x^{m} \in S_{\beta}$ for some $m>0$, as $A$ is archimedean and $S_{\beta}$ is an ideal of $T\left(S_{\beta}\right)$. Therefore, we would have $x^{m} \in S_{\alpha} \cap S_{\beta}$, another contradiction. Thus $|\Gamma|=2$.

Hence we will let $S=S_{0} \cup S_{1}$ where $S_{0}$ is a power-joined $N$ - 
semigroup and $S_{1}$ is isomorphic to a subgroup of the group of permutation translations of $S_{0}$

By $L_{n}\left(S_{0}, S_{1}\right)$ we mean the collection of nil-congruences on $S_{0}$ which extend to congruences on $S$ (i.e., $\sigma \in L_{n}\left(S_{0}, S_{1}\right)$ if $\sigma$ is a nil-congruence on $S_{0}$ and $(a, b) \in \sigma$ and $x \in S_{1}$ implies $\left.(a x, b x) \in \sigma\right)$. Similarly $L_{r}\left(S_{0}, S_{1}\right)$ is the collection of Rees congruences on $S_{0}$ which extend to congruences on $S$. The next lemma is a generalization of Lemma 2.4.

LEMMA 3.3. Let $S=S_{0} \cup S_{1}$ be a commutative semigroup with $S_{0}$ an ideal which is an $N$-semigroup. If $L(S)$ is a modular lattice then $L_{n}\left(S_{0}, S_{1}\right)=L_{r}\left(S_{0}, S_{1}\right)$.

Proof. Let $\sigma \in L_{n}\left(S_{0}, S_{1}\right) \backslash L_{r}\left(S_{0}, S_{1}\right)$. Let $J$ be the $\sigma$-class of $S_{0}$ corresponding to the zero of $S_{0} / \sigma . \quad J$ is an ideal of $S_{0}$ which is an ideal of $S$. Hence the Rees congruence $\bmod J, \rho_{J}$, is in $L_{r}\left(S_{0}, S_{1}\right)$. Let $\tau$ be the group congruence on $S_{0}$ determined by the natural homomorphism of $S_{0}$ onto one of its structure groups $G$. Then $\bar{\sigma}=$ $\sigma \cup \iota_{S_{1}}, \bar{\rho}=\rho_{J} \cup \iota_{S_{1}}$ are in $L(S)$. Let $\bar{\tau}$ be the unique group congruence on $S$ extending $\tau$. Then by an argument similar to that used in Lemma 2.4 we see that $L(S)$ contains

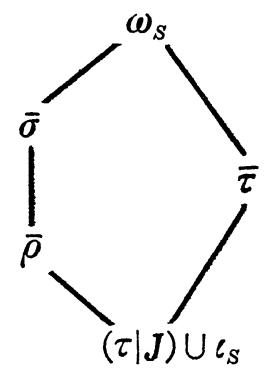

Hence $L(S)$ is not modular.

Hall [7] and Dickinson [4] gave the following characterization of $T(S)$ where $S=(G, I)$ is an $N$-semigroup.

$$
T(S)=\left\{[m, g] \in N^{0} \times G: m+I(g, h)-1 \geqq 0 \text { for all } h \in G\right\} \text {. }
$$

The action of an element $[m, g] \in T(S)$ on an element $(n, h) \in S$ is

$$
[m, g](n, h)=(m+n+I(g, h)-1, g h) .
$$

The group of units $T_{u}(S)$ of $T(S)$ is shown by Dickinson to be

$$
T_{u}(S)=\left\{[0, g] \in N^{0} \times G: I(g, h)=1 \text { for all } h \in G\right\} .
$$


with an identity element. Then $L(S)$ is a modular lattice if and only if $S$ is an abelian group or a nonnegative cone of an additive subgroup of the rationals.

Proof. Assume $L(S)$ is a modular lattice, and assume that $S$ is not an abelian group. Then, as we noted above, $S=S_{0} \cup S_{1}$ where $S_{0}$ is a power-joined $N$-semigroup ideal of $S$ and $S_{1}$ is an abelian group. Suppose that $S_{0}=(G, I)$. From previous remarks we can consider $S_{1}$ to be a subgroup of $T_{u}\left(S_{0}\right)$. Define a relation $\sigma$ on $S_{0}$ by

$$
((m, g),(n, h)) \in \sigma \text { if and only if }\left\{\begin{array}{l}
m>0 \text { and } n>0, \text { or } \\
m=n=0 \text { and }[0, k](m, g)=(n, h) \\
\text { for some }[0, k] \in S_{1} .
\end{array}\right.
$$

Then $\sigma \in L_{n}\left(S_{0}, S_{1}\right)$ and if $S_{1}$ is not the trivial group then $\sigma \notin L_{r}\left(S_{0}, S_{1}\right)$. Hence by Lemma 3.3 $S_{1}$ must be the trivial group. Since $S_{1}$ is trivial, every congruence on $S_{0}$ extends to a congruence on $S$. Therefore $L\left(S_{0}\right)$ is a modular lattice, also; so by Theorem $2.11 S_{0}$ is a positive cone of a rational group. Therefore $S$ is a nonnegative cone of a rational group.

To see the converse, consider the following. Let $S=S_{0} \cup\{1\}$ be a power-joined $N$-semigroup $S_{0}$ with an identity 1 adjoined. Define a map $F$ of $L(S)$ into the direct product of $L\left(S_{0}\right)$ and the two element lattice $2=\{0,1\}$, with $0>1$, by

$$
F(\sigma)=\left\{\begin{array}{lll}
\left(\sigma \mid S_{0}, 1\right) & \text { if } \quad 1_{\sigma}=\{1\} \\
\left(\sigma \mid S_{0}, 0\right) & \text { if } \quad 1_{\sigma} \neq\{1\}
\end{array}\right.
$$

where $1_{\sigma}$ denotes the $\sigma$-class containing 1 .

$F$ preserves joins. Let $\sigma, \tau \in L(S)$.

Case (1). $1_{\sigma}=\{1\}=1_{\tau}$. Then $F(\sigma) \vee F(\tau)=\left(\sigma \mid S_{0}, 1\right) \vee\left(\tau \mid S_{0}, 1\right)=$ $\left(\sigma\left|S_{0} \vee \tau\right| S_{0}, 1\right)$ and $F(\sigma \vee \tau)=\left((\sigma \vee \tau) \mid S_{0}, 1\right)$. And $(\sigma \vee \tau) \mid S_{0}=$ $\sigma\left|S_{0} \vee \tau\right| S_{0}$ is clear. Thus $F(\sigma) \vee F(\tau)=F(\sigma \vee \tau)$.

Case (2). $1_{\sigma}=\{1\}$ and $1_{\tau} \neq\{1\}$. Then $F(\sigma) \vee F(\tau)=\left(\sigma \mid S_{0}, 1\right) \vee$ $\left(\tau \mid S_{0}, 0\right)=\left(\sigma\left|S_{0} \vee \tau\right| S_{0}, 0\right)$. Since $1_{\tau} \neq\{1\}$ and $\tau \subseteq \sigma \vee \tau$ we have $1_{\sigma \vee \tau} \neq\{1\}$ so $F(\sigma \vee \tau)=\left((\sigma \vee \tau) \mid S_{0}, 0\right)$. Thus to show $F(\sigma) \vee F(\tau)=F(\sigma \vee \tau)$ we need only show that $(\sigma \vee \tau)\left|S_{0}=\sigma\right| S_{0} \vee \tau \mid S_{0}$. Inclusion of $\sigma\left|S_{0} \vee \tau\right| S_{0}$ in $(\sigma \vee \tau) \mid S_{0}$ is clear. We therefore choose $(a, b) \in(\sigma \vee \tau) \mid S_{0}$. Then there exist $a=a_{0}, a_{1}, \cdots, a_{n}=b$ in $S$ such that $\left(a_{i}, a_{i+1}\right) \in \sigma \cup \tau$ for all $i=0,1, \cdots, n-1$. Since $1_{\sigma}=\{1\}$, if $a_{i_{0}}=1$ for some $i_{0}$ then we can delete $a_{i_{0}}$ from the sequence and still have a chain of $\sigma \cup \tau$-related 
elements between $a$ and $b$ all of which will now lie in $S_{0}$. Hence $(a, b) \in \sigma\left|S_{0} \vee \tau\right| S_{0}$ and so $(\sigma \vee \tau)\left|S_{0} \leqq \sigma\right| S_{0} \vee \tau \mid S_{0}$ and we have equality of $F(\sigma \vee \tau)$ and $F(\sigma)<F(\tau)$ in this case.

Case (3). $1_{\sigma} \neq\{1\}$ and $1_{\tau} \neq\{1\}$. Hence $1_{\sigma \vee \tau} \neq\{1\}$. Therefore to show that $F(\sigma \vee \tau)=F(\sigma) \vee F(\tau)$ we need only show that $(\sigma \vee \tau) \mid S_{0} \subseteq$ $\sigma\left|S_{0} \vee \tau\right| S_{0}$ as in the last case. Let $(a, b) \in(\sigma \vee \tau) \mid S_{0}$. Then as before there exists $a=a_{0}, a_{1}, \cdots, a_{n}=b$ in $S$ such that $\left(a_{i}, a_{i+1}\right) \in$ $\sigma \cup \tau$ for each $i=0,1, \cdots, n-1$. As in case (2) we wish to lift this to a chain of $\sigma \cup \tau$-related elements in $S_{0}$ from $a$ to $b$. To accomplish this note that $\sigma$ and $\tau$ are group congruences on $S$ and hence on $S_{0}$. And by Theorem $2.2 \sigma\left|S_{0} \cap \tau\right| S_{0}$ is also a group congruence on $S_{0}$. Choose $u \in S_{0}$ such that $u_{\left(\sigma\left|S_{0} \cap \tau\right| S_{0}\right)}$ is the identity element of $S_{0} /\left(\sigma\left|S_{0} \cap \tau\right| S_{0}\right)$. Then $u_{\sigma}$ is the identity of $S / \sigma$ and $u_{\tau}$ is the identity of $S / \tau$. So we have $\left(a, a_{0} u\right),\left(a_{i} u, a_{i+1} u\right)$ for $i=0,1$, $\cdots, n-1$, and $\left(a_{n} u, b\right)$ are all in $\sigma \cup \tau$. Thus $(a, b) \in \sigma\left|S_{0} \vee \tau\right| S_{0}$, and we are done. Thus $F$ preserves joins.

$F$ preserves intersections. For any two congruences $\sigma, \tau \in L(S)$ we always have $(\sigma \cap \tau)\left|S_{0}=\sigma\right| S_{0} \cap \tau \mid S_{0}$. Also, if $1_{\sigma}=\{1\}$ or $1_{\tau}=\{1\}$ then $1_{o \cap \tau}=\{1\}$. It also follows from the remarks about finding the element $u$ in case (3) above that if $1_{\sigma} \neq\{1\}$ and $1_{\tau} \neq\{1\}$ then $1_{\sigma \cap \tau} \neq$ $\{1\}$. Hence $F$ preserves intersections.

$F$ is one-to-one. Let $\sigma, \tau \in L(S)$. If $F(\sigma)=F(\tau)$ and $F(\sigma)=$ $\left(\sigma \mid S_{0}, 1\right)$ and $F(\tau)=\left(\tau \mid S_{0}, 1\right)$ then $\sigma\left|S_{0}=\tau\right| S_{0}$, but in this case $\sigma=\sigma \mid S_{0} \cup\{(1,1)\}$ and $\tau=\tau \mid S_{0} \cup\{(1,1)\}$. Therefore $\sigma=\tau$. If $F(\sigma)=$ $F(\tau)$ and $F(\sigma)=\left(\sigma \mid S_{0}, 0\right)$ and $F(\tau)=\left(\tau \mid S_{0}, 0\right)$ then $\sigma$ and $\tau$ are group congruences on $S$ which are the unique extensions of $\sigma \mid S_{0}$ and $\tau \mid S_{0}$ to group congruences on $S$. Thus $\sigma\left|S_{0}=\tau\right| S_{0}$ implies that $\sigma=\tau$. And we therefore see that $F$ is one-to-one.

The above shows that $F$ is a closed lattice isomorphism of $L(S)$ into $L\left(S_{0}\right) \times 2$.

To complete the proof of the converse of Theorem 3.4 let $S$ be a nonnegative cone of a subgroup of the additive rationals. Then $S=S_{0} \cup\{0\}$ where $S_{0}$ is the positive part of $S . S_{0}$ is a power-joined $N$-semigroup and by Theorem $2.11 L\left(S_{0}\right)$ is a modular lattice. Thus applying the map $F$ to this $S$ shows that $L(S)$ is also a modular lattice. This completes the proof of the theorem.

4. Summary and further observations. So far we have shown

THEOREM 4.1. The commutative cancellative semigroups whose 
congruence lattice is a modular lattice are

(1) The abelian groups.

and

(2) The positive cones of subgroups of the additive rationals,

(3) The nonnegative cones of subgroups of the additive rationals.

Consider the following example. Let $X$ be an index set and let $D_{1}$ be the direct product of $|X|$ copies of $N^{0}$, and let $D=\{n=$ $\Pi_{x} n_{x} \in D_{1}$ : there exists $M_{n}>0$ such that $n_{x}<M_{n}$ for all $\left.x \in X\right\}$. Then $D$ is a commutative cancellative semigroup and the greatest semilattice homomorphic image of $D$ is the semilattice (actually lattice) $2^{X}$ of all subsets of $X$. Since every semilattice can be embedded into $2^{X}$ for some set $X$, we see that every semilattice is a homomorphic image of some commutative cancellative semigroup. In [6] R. Freese and J. B. Nation showed that the collection of congruence lattices of semilattices satisfies no nontrivial lattice identity. From this and the remarks above we see the following theorem.

THEOREM 4.2. The collection of congruence lattices of commutative cancellative semigroups satisfies no nontrivial lattice identity.

In [3] R. A. Dean and R. H. Oehmke and in [18] T. Tamura and W. Etterbeek showed that the lattice of congruences on a locally cyclic semigroup is a distributive lattice. Also, a theorem (see [12]) due to Ore gives the groups whose subgroup lattice is a distributive lattice to be precisely the locally cyclic groups. From these results and the fact that a distributive lattice is a modular lattice we have immediately from Theorem 4.1 the following

THEOREM 4.3. The commutative cancellative semigroups whose congruence lattice is distributive are

(1) The locally cyclic abelian groups.

(2) The positive cones of subgroups of the additive rationals.

(3) The nonnegative cones of subgroups of the additive rationals.

Noticing the result of the theorem we can easily obtain that Ore's theorem almost holds for commutative cancellative semigroups. That is, we have

CoROLlaRY 4.4. Let $S$ be a commutative cancellative semigroup. Then $L(S)$ is distributive if and only if $S$ is locally cyclic or $S^{1}$ is locally cyclic with an identity adjoined. ( $S^{1}$ equals $S$ if $S$ already 
has an identity element and $S^{1}$ is $S$ with an identity adjoined if $S$ has no identity.)

Proof. Let $S$ be a locally cyclic commutative cancellative semigroup. Then for all $x, y \in S$ there exist $z \in S$ and $m, n \in N$ such that $x=z^{m}$ and $y=z^{n}$. Thus $x^{n}=y^{m}$, and $x y^{-1}$ or $y x^{-1}$ is in $S$. Hence if $S$ is not a group then $S$ is a power-joined $N$-semigroup in which divides is a total order. That is, $S$ is a positive cone of a rational group. The proof is now easily finished by looking at the theorem, since positive cones of rational groups are locally cyclic semigroups.

\section{REFERENCES}

1. A. H. .Clifford and G. B, Preston, The Algebraic Theory of Semigroups, Vol. 1, Amer. Math. Soc., Providence, Rhode Island, 1961.

2. A. H. Clifford and G. B. Preston, The Algebraic Theory of Semigroups, Vol. 2, Amer. Math. Soc., Providence, Rhode Island, 1967.

3. R. A. Dean and R. H. Oehmke, Idempotent semigroups with distributive right congruence lattices, Pacific J. Math., 14 (1964), 1187-1209.

4. R. P. Dickinson, Right zero unions of semigroups, Dissertation, University of Calif., Davis, 1970.

$5 . \quad$ Noncancellative congruences on $\mathrm{N}$-semigroups, Proc. Amer. Math. Soc., 36: 2 (1972), 317-325.

6. R. Freese, and J. B. Nation, Congruence lattices of semilattices, Pacific J. Math., 49 (1973), 51-58.

7. R. Hall, The structure of certain commutative separative and commutative cancellative semigroups, Dissertation, Pennsylvania State University, 1969.

8. H. B. Hamilton, Congruences on $N$-semigroups, Pacific J. Math., 75 (1978), 423-448.

9. H. B. Hamilton, T. E. Nordahl and T. Tamura, Commutative cancellative semigroups without idempotents, Pacific J. Math., 61 (1975), 441-456.

10. E. Hewitt and H. S. Zuckermann, The $l_{1}$ algebra of a commutative semigroup, Trans. Amer. Math. Soc., 83 (1956), 70-97.

11. Y. Kobayashi, Homomorphisms on $N$-semigroups into $R_{+}$and the structure of $N$ semigroups, J. Math., Tokushima Univ., 7 (1973), 1-20.

12. O. Ore, Structures and group theory I-II, Duke Math. J., 3 (1937), 149-174; 4 (1938), 247-269.

13. M. Petrich, Introduction to Semigroups, C. E. Merril Publ. Co., Columbus, Ohio, 1973.

14. T. Tamura, Commutative nonpotent archimedean semigroups with cancellation law I, J. Gakugei, Tokushima Univ., VIII (1957), 5-11.

15. —, Irreducible $N$-semigroups, Math. Nachr., 63 (1974), 71-88.

16. - Basic study of $\mathrm{N}$-semigroups and their homomorphisms, Semigroup Forum, 8 (1974), 21-50.

17. - Commutative archimedean cancellative semigroups withoup idempotent, Seminaire Duheil-Pisot (Algebre et Theorie des nombres) $23^{\circ}$ annee 1969/70, Demigroupes, $\mathrm{n}^{\circ} 5,19 \mathrm{p}$.

18. T. Tamura and W. Etterbeek, The lattice of congruences of locally cyclic semigroups, Proc. Japan Acad., 42, No. 7 (1966), 682-684.

19. T. Tamura and H. B. Hamilton, The study of commutative semigroups with greatest group-homomorphisms, Trans. Amer. Math. Soc., 173 (1972), 401-419.

20. T. Tamura and M. Sasaki, Positive rational semigroups and commutative power- 
joined cancellative semigroups without idempotent, Czech. Math. J., 21 (96), (1971), 567-576.

Received April 29, 1976 and in revised form September 21, 1977. Some of these results were presented at the San Francisco meeting of the AMS in January 1974 and were announced in [Notices AMS 21 (1974), A-92]. Theorem 4.3 was obtained by M. P. Dorofeeva in 1975 [Summaries of Talks, All-Union Alg. Sympos., Gomel, 1975, Part I, 208 (Russian)].

California State University

SaCramento, CA 95819 



\section{PACIFIC JOURNAL OF MATHEMATICS}

\section{EDITORS}

RichaRd ARENS (Managing Editor)

University of California

Los Angeles, CA 90024

Charles W. Curtis

University of Oregon

Eugene, OR 97403

C. C. Moore

University of California

Berkeley, CA 94720

\section{J. DUGUNDJI}

Department of Mathematics

University of Southern California

Los Angeles, CA 90007

R. FinN and J. Milgram

Stanford University

Stanford, CA 94305

\section{E. F. BECKENBACH \\ B. H. NeumanN

\author{
UNIVERSITY OF BRITISH COLUMBIA \\ CALIFORNIA INSTITUTE OF TECHNOLOGY \\ UNIVERSITY OF CALIFORNIA \\ MONTANA STATE UNIVERSITY \\ UNIVERSITY OF NEVADA, RENO \\ NEW MEXICO STATE UNIVERSITY \\ OREGON STATE UNIVERSITY \\ UNIVERSITY OF OREGON
}

F. WOLF

K. Yoshida

\section{SUPPORTING INSTITUTIONS}

\author{
UNIVERSITY OF SOUTHERN CALIFORNIA \\ STANFORD UNIVERSITY \\ UNIVERSITY OF HAWAII \\ UNIVERSITY OF TOKYO \\ UNIVERSITY OF UTAH \\ WASHINGTON STATE UNIVERSITY \\ UNIVERSITY OF WASHINGTON
}

The Supporting Institutions listed above contribute to the cost of publication of this Journal, but they are not owners or publishers and have no responsibility for its content or policies.

Mathematical papers intended for publication in the Pacific Journal of Mathematics should be in typed form or offset-reproduced, (not dittoed), double spaced with large margins. Please do not use built up fractions in the text of the manuscript. However, you may use them in the displayed equations. Underline Greek letters in red, German in green, and script in blue. The first paragraph or two must be capable of being used separately as a synopsis of the entire paper. Items of the bibliography should not be cited there unless absolutely necessary, in which case they must be identified by author and journal, rather than by item number. Manuscripts, in triplicate, may be sent to any one of the editors. Please classify according to the scheme of Math. Reviews, Index to Vol. 39. All other communications should be addressed to the managing editor, or Elaine Barth, University of California, Los Angeles, California, 90024.

50 reprints to each author are provided free for each article, only if page charges have been substantially paid. Additional copies may be obtained at cost in multiples of 50 .

The Pacific Journal of Mathematics is issued monthly as of January 1966. Regular subscription rate: $\$ 72.00$ a year (6 Vols., 12 issues). Special rate: $\$ 36.00$ a year to individual members of supporting institutions.

Subscriptions, orders for numbers issued in the last three calendar years, and changes of address should be sent to Pacific Journal of Mathematics, P.O. Box 969, Carmel Valley, CA 93924, U.S.A. Older back numbers obtainable from Kraus Periodicals Co., Route 100, Millwood, NY 10546.

PUBLISHED BY PACIFIC JOURNAL OF MATHEMATICS, A NON-PROFIT CORPORATION

Printed at Kokusai Bunken Insatsusha (International Academic Printing Co., Ltd.). 8-8, 3-chome, Takadanobaba, Shinjuku-ku, Tokyo 160, Japan. 


\section{Pacific Journal of Mathematics \\ Vol. 79, No. $2 \quad$ June, 1978}

David R. Adams, Quasi-additivity and sets of finite $L^{p}$-capacity ........ 283

George M. Bergman and Warren Dicks, Universal derivations and universal

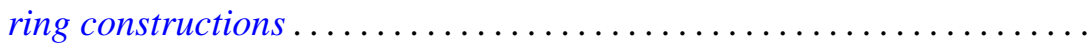

Robert F. Brown, Addendum to: "Fixed points of automorphisms of compact Lie groups".........................................

Eugene Frank Cornelius, Jr., Characterization of a class of torsion free

groups in terms of endomorphisms .......................

Andres del Junco, A simple measure-preserving transformation with trivial centralizer..................................... 357

Allan Lee Edmonds, Extending a branched covering over a handle ...... 363

Sjur Flam, A characterizaton of $\mathbf{R}^{2}$ by the concept of mild convexity .......

Claus Gerhardt, $L^{p}$-estimates for solutions to the instationary Navier-Stokes

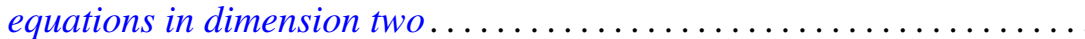

Kensaku Gomi, Finite groups with a standard subgroup isomorphic to

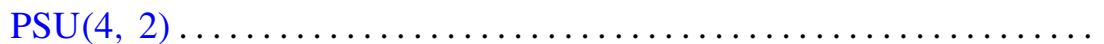

E. E. Guerin, A convolution related to Golomb's root function ........... 463

$\mathrm{H}$. B. Hamilton, Modularity of the congruence lattice of a commutative cancellative semigroup .................................

Stephen J. Haris, Complete reducibility of admissible representations over function fields.

Shigeru Itoh and Wataru Takahashi, The common fixed point theory of singlevalued mappings and multivalued mappings... ...

James E. Joseph, Multifunctions and graphs .............

Bruce Magurn, Images of $S K_{1} Z G$. .

Arnold Koster Pizer, A note on a conjecture of Hecke... .

Marlon C. Rayburn, Maps and h-normal spaces ...........

Barada K. Ray and Billy E. Rhoades, Corrections to: "Fixed-point theorems for mappings with a contractive iterate" ..............

Charles Irvin Vinsonhaler, Corrections to: "Torsion free abelian groups quasiprojective over their endomorphism rings. II". . . 